\title{
Erratum to: Hepatic hydatid disease complications: review of imaging findings and clinical implications
}

\author{
Khalefa Ali Alghofaily, ${ }^{1}$ Mnahi Bin Saeedan, ${ }^{2}$ Ibtisam Musallam Aljohani, ${ }^{3}$ \\ Mohannad Alrasheed, ${ }^{4}$ Sebastian McWilliams, ${ }^{5}$ Abdullah Aldosary, ${ }^{6}$ \\ Mohammed Neimatallah ${ }^{4}$ \\ ${ }^{1}$ Medical Imaging Department, College of Medicine, Al-Qassim University, Buraydah, Saudi Arabia \\ ${ }^{2}$ Department of Radiology, MBC-28, King Faisal Specialist Hospital and Research Center, P.O. Box 3354, Riyadh 11211, Saudi \\ Arabia \\ ${ }^{3}$ Medical Imaging Department, Ministry of National Guard, Health Affairs, King Abdulaziz Medical City, Riyadh, Saudi Arabia \\ ${ }^{4}$ Department of Radiology, King Faisal Specialist Hospital and Research Center, Riyadh, Saudi Arabia \\ ${ }^{5}$ Mallinckrodt Institute of Radiology, Washington University, St. Louis, USA \\ ${ }^{6}$ Department of Radiology, King Fahad Medical City, Riyadh, Saudi Arabia
}

\section{Erratum to: Abdom Radiol (2016) DOI 10.1007/s00261-016-0860-2}

The original version of this article unfortunately contained a mistake. The first author's name "Alghofaily Ali Khalefa" should read as "Khalefa Ali Alghofaily". It is now corrected.

The online version of the original article can be found under doi: 10.1007/s00261-016-0860-2. 